\title{
Personalized Rehabilitation Recognition Model upon ANFIS
}

\author{
Yao-Chiang Kan ${ }^{1}$, Yu-Chieh Kuo ${ }^{2}$, Hsueh-Chun Lin ${ }^{2, *}$ \\ ${ }^{1}$ Department of Communication Engineering, Yuan Ze University, Taoyuan, Taiwan \\ ${ }^{2}$ Institute of Health Service Administrations, China Medical University, Taoyuan, Taiwan \\ Received 13 March 2019; received in revised form 19 May 2019; accepted 11 July 2019
}

DOI: https://doi.org/10.46604/peti.2020.3912

\begin{abstract}
This study applied the Adaptive Neuro-Fuzzy Inference System (ANFIS) to design a recognition model of personalized rehabilitation. In the model, the user may take a wearable sensor and follow the assigned joint-relax exercise to measure the motions of the upper limbs. The sensor that is embedded with the chips of accelerometer, gyroscope, and inclinometer produced the sample datasets due to the exercise schedule of physiotherapy assignment. All motion samples were labeled by arbitrary numbers, which can be identified to the specific motion, for the data training process. A Fuzzy Inference System (FIS) was initially designed by the steps of data pre-processing, featuring, fuzzifying, and ruling Fuzzy logics according to the sample datasets. The FIS was then trained by the ANFIS for optimization by tuning parameters of the features. In testing, the accomplished FIS could recognize the motion features by the defuzzifier that infers the label corresponding to the motion. As a result, the average recognition rate was higher than $90 \%$ when the testing motions followed the sampling schedule of the physiotherapy assignment. The model can be applied in the ubiquitous healthcare measurement for health services. The professionals can assess whether the subject obeyed the assigned program or not based on detail motions of the exercise. This approach can be enabled on the trackable interface for the physiatrists to screen the motions of routine rehabilitation.
\end{abstract}

Keywords: ANFIS, physiotherapy, rehabilitation recognition, sensor-enabled wristband, ubiquitous healthcare

\section{Introduction}

The physiotherapy provides the rehabilitation program for the patients who are weakened on motions. The therapeutic exercise is thus designed to flex the joints of their injured limbs. The physical therapists scheme the rehabilitation programs including the joint kinematics for those disabled parts, e.g. flexion, extension, abduction, adduction, pronation, supination, rotation, and deviation [1]. Many clinical studies of healthcare services advised that the routine exercises with a scheduled rehabilitation period are helpful in healing [2]. The physiatrists request daily practice at home, or with facilities in the hospital, for the patients and observe their performance to adjust the health promotion program of joint motions.

The modern healthcare services with internet technique offered Ubiquitous Healthcare Measurement (UHM) for patients to support self-management for homely rehabilitation. The physiatrists are able to screen the daily records of the patients via the UHM for evaluating whether the assigned exercise needs progressive revision or not [3]. The UHM enables rehabilitation in a wireless area network by wearing non-imaging monitoring devices to record the motion data of patients with privacy [4-5]. Therefore, a wristband with accelerometer and gyroscope was widely used to detect human activities such as housekeeping, falling, walking, and sporting in recent years [6]. With Bluetooth technology, the sensor wristband can efficiently transport the measurement data to a smartphone for the UHM. The personalized rehabilitation data can be measured by a Bluetooth sensor and be recognized by the proper algorithms for management [7-8].

* Corresponding author. E-mail address: snowlin@mail.cmu.edu.tw 
For the study of activity recognition, the algorithms involving Artificial Neural Network (ANN), Backward Propagation Neural Network (BPNN), Fuzzy logic theory, etc. are popular for computation. The rule- based Fuzzy Inference System (FIS) is quite appropriate for the tendency of stationary motions (e.g. rehabilitation) [9]. A Fuzzy set involves Membership Funcions (MFs) to fuzzify the input and output features; then, the logic rules based on if- then syntax are scripted to defuzzify the MFs for inference (i.e. defuzzification) [10-11]. In the FIS, the Mamdani and Sugeno models perform two MF types, which the MFs of output features are respectively formatted by geometrical-shape functions and linear-expression crisps [12-13]. The Adaptive Neuro- Fuzzy Inference System (ANFIS) enables a data training process to adjust the FIS parameters for improving the inference capability [14]. In general, the FIS was suggested to recognize the inferable features of the rehabilitation exercises in the UHM.

This study suggested a prototype of the rehabilitation model, which applied ANFIS to recognize the personalized motions. A wearable sensor compliant with Bluetooth protocol was employed to collect data. The ANFIS enables an adaptive data training process based on the Fuzzy logic rules to achieve personalized recognition.

\section{Methodology}

The proposed model requests the ANFIS to recognize the rehabilitation exercises. The user wears a Bluetooth-compliant sensor JY-61 that embeds the chips of accelerometer, gyroscope, and inclinometer into the device to detect 3-axis components of accelerations, angular velocities, and tilt angles, respectively.
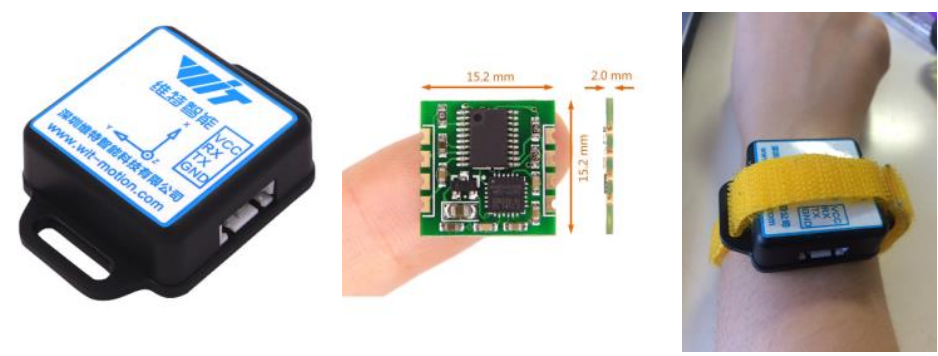

(a) The wearable JY-61 sensor, embedded chips, and wearing location on the wrist
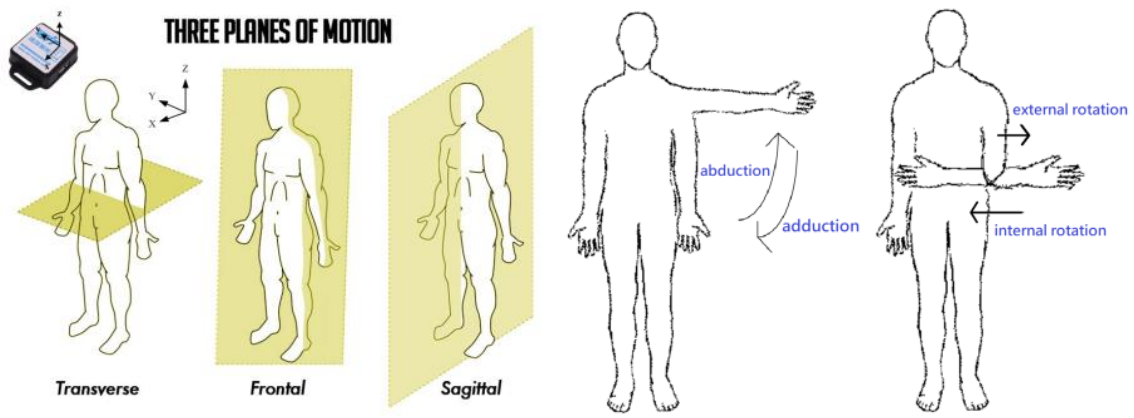

(b) Definition of the local and global coordinates for wearing the sensor, and the joint-relaxing motions of abduction-adduction and external-internal rotation

Fig. 1 Prepared instrument and exercises for the personalized rehabilitation recognition

\subsection{Exercise design}

We took three rehabilitation exercises as an example, in which the user wore the sensor on the wrist in the measurement. The sensor was able to send 100 datasets of signals per second. As shown in Fig. 1, the neutral position of the human body is the global coordinate, and the center of the sensor is the local coordinate. The motions of the exercise were designed by global coordinates, but the sensor signals were measured in local coordinates. When the exercise was activating, the sensor data were corresponding to the initial position of the sensor. Two exercises including abduction, adduction, and rotation of the shoulder and elbow were applied to measure the range of motion regarding the upper-limb joints. For the exercises addressed below, the sensor was worn on the wrist and each exercise cycle was repeated five times. 
(1) Shoulder Ex. with horizontal abduction and adduction (abd-add) motions - the arm initially straightens downward and the palm faces inward: keep the initial state for two seconds $\rightarrow$ raise the arm up laterally to shoulder level (abduction) in four seconds $\rightarrow$ hold the limb at shoulder level for two seconds $\rightarrow$ drop the arm down to the initial state in four seconds.

(2) Elbow Ex. with external and internal rotation (ext-int rot) motions - the elbow bends to $90^{\circ}$ and the palm faces inward the abdomen, and the upper arm must close to the body: keep the initial state for two seconds $\rightarrow$ rotate the arm away from facing inward toward the abdomen to facing outward in four seconds $\rightarrow$ hold the limb there for two seconds $\rightarrow$ rotate the arm toward the abdomen in four seconds.

\subsection{ANFIS process}

A typical ANFIS involves five parts in a cyclical process: (a) data preprocessing, (b) feature visualizing, (c) fuzzifying, (d) data training, and (e) defuzzifying; in which, the process trains the FIS to obtain the optimized parameters based on the Fuzzy logic rules for recognizing the personalized rehabilitation motions. The flowchart of the ANFIS process is shown in Fig. 2.

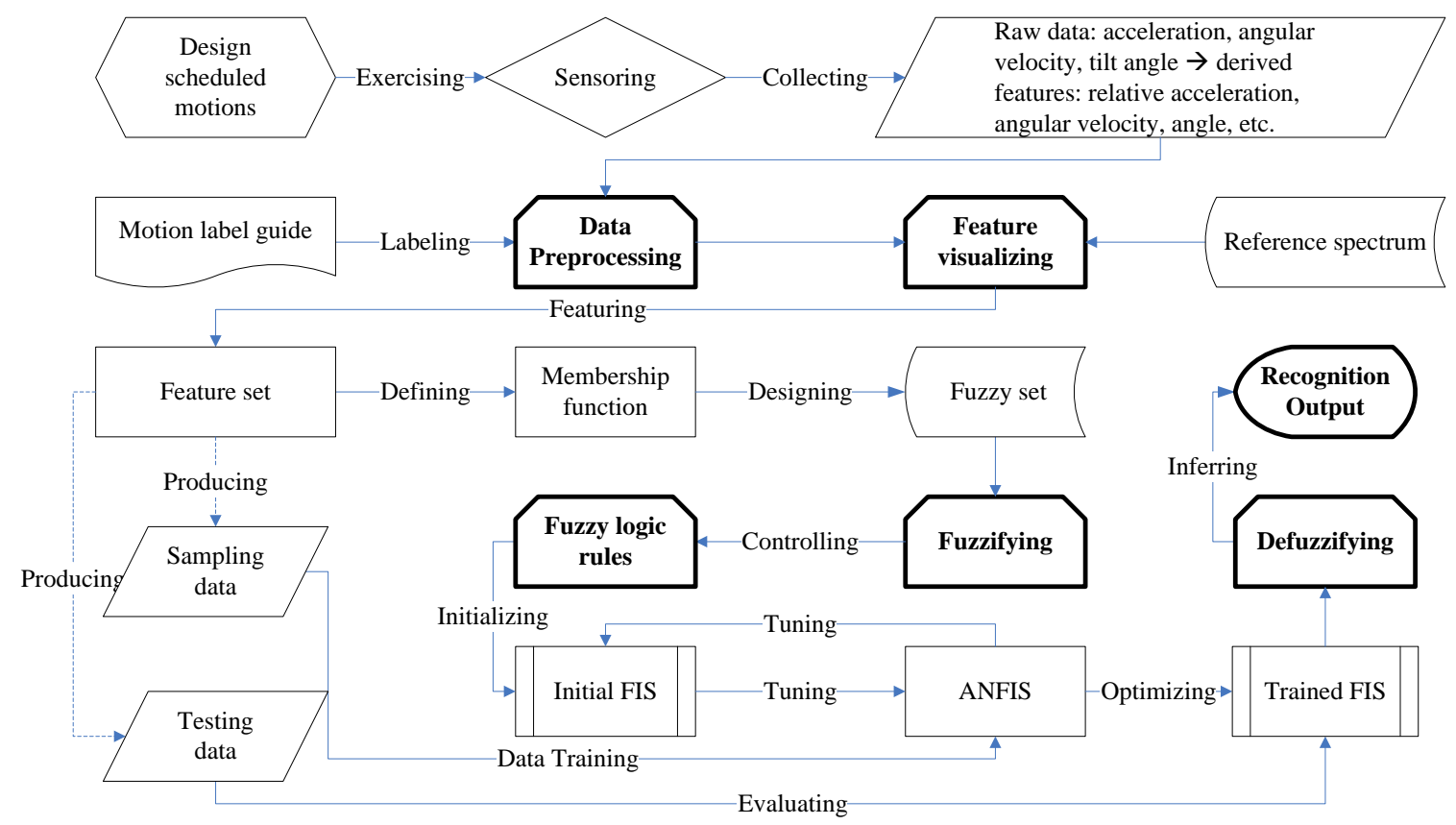

Fig. 2 Machine learning flowchart of ANFIS for recognizing the rehabilitation exercise

(a) The user needed to exactly obey the motion schedule of the designed exercise. The sample datasets were sent by the wearable sensor and we were able to define the motion label with the arbitrary numbers in a specific range. For the example of the shoulder exercise, the motions were labeled by random numbers in $[4,5),[5,6),[6,7)$, and $[7,8)$, respectively. Then, the user practiced the same schedule to prepare a test dataset for evaluation. In this study, the possible features include relative acceleration, angular velocity, and tilt angle of the motion with respect to the initial sensor position (i.e. rACC, rANGV, and rANG for starting moving, in moving, and limb position, respectively).

(b) We observed the accessible features according to the diagrams of senor data variation as shown in Fig. 3; whereas, the time-domain data distribution was transformed into the frequency-domain spectrum. The spectra of the available features are related to the Membership Function (MF) of the corresponding motions in Fuzzy computing. For example, we selected rANGx and rANGVx (i.e. the components in x-axis) as the features to perform the still and moving motions for the abd-add exercise.

(c) We created the FIS by choosing the proper MFs for the Fuzzy set that presents a featuring process before data training. As shown in Fig. 3 for example, we adopted the triangle and trapezoid MFs for the input features, and four triangle MFs for the output features according to the spectra of rANGVx and rANGx variables in the abd-add motions. The MFs of output feature is corresponding to the labels defined in the previous four-step motions. Combination of the various input features 
with the proper MFs can yield a Fuzzy set available for the same output motions. The MFs of output feature, which is initially designed as the Mamdani-type function, must be converted to the Sugeno-type crisp set before the training process in the ANFIS. Besides, the amount of the output crisps for the Sugeno-type Fuzzy set must be a multiplication of those in the input features.

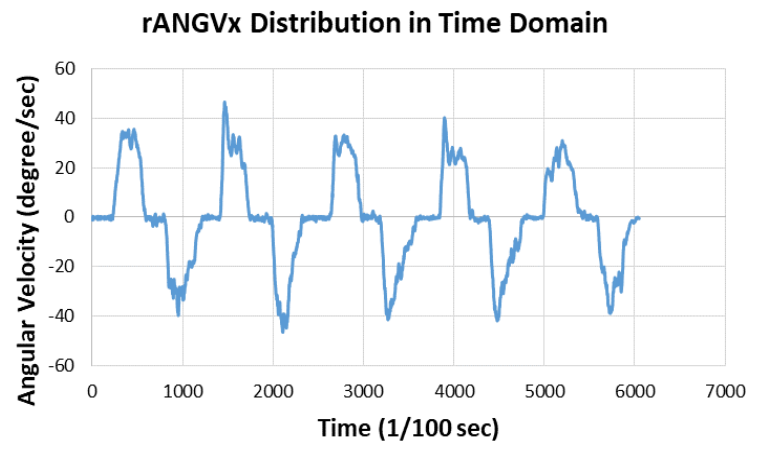

(a) The feaure rANGVx in time-domain distribution

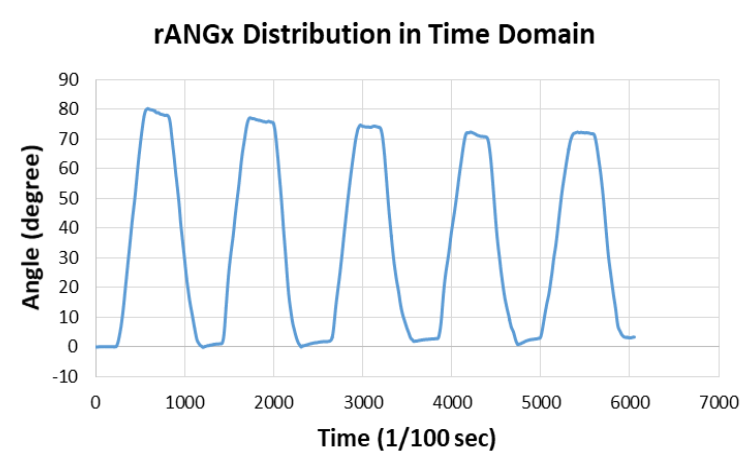

(c) The feaure rANGx in time-domain distribution

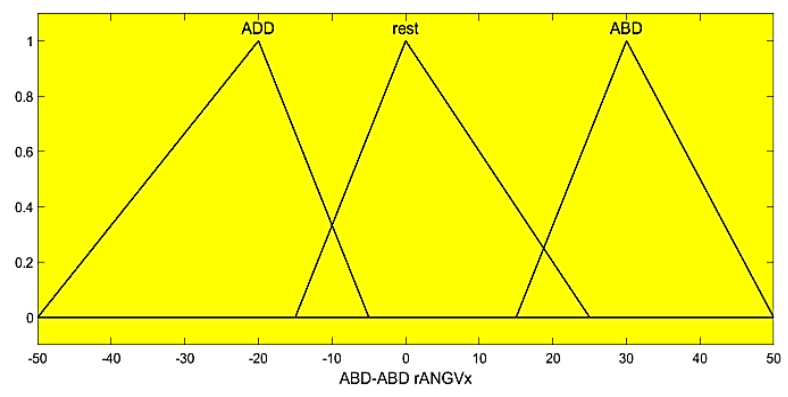

(e) The MFs of rANGVx feature

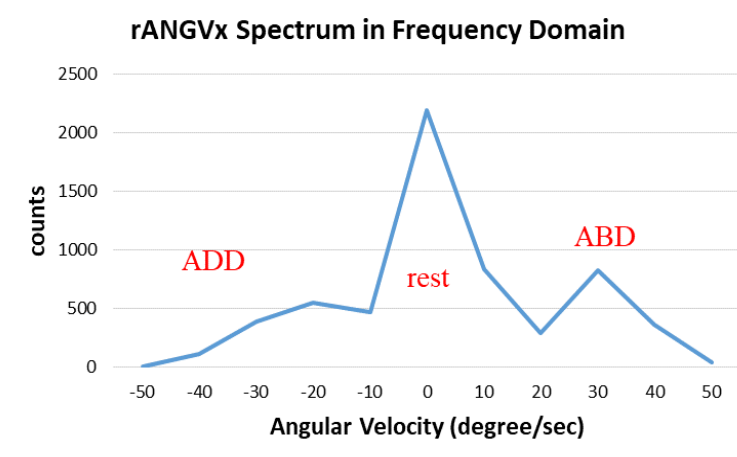

(b) The feature rANGVs in frequency-domain spectrum

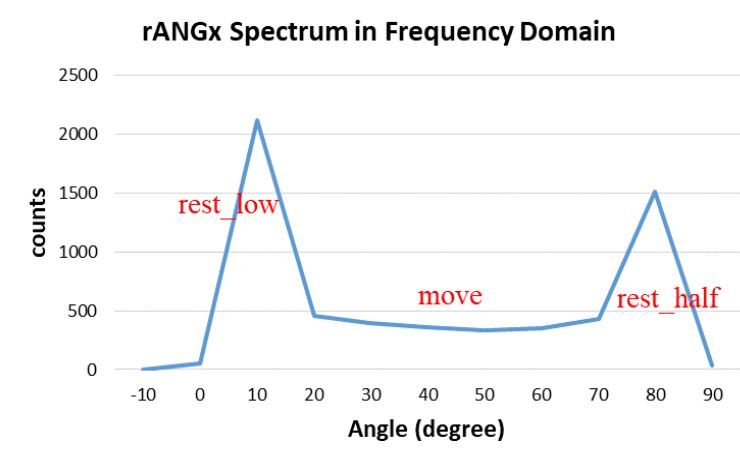

(d) The feature rANGs in frequency-domain spectrum

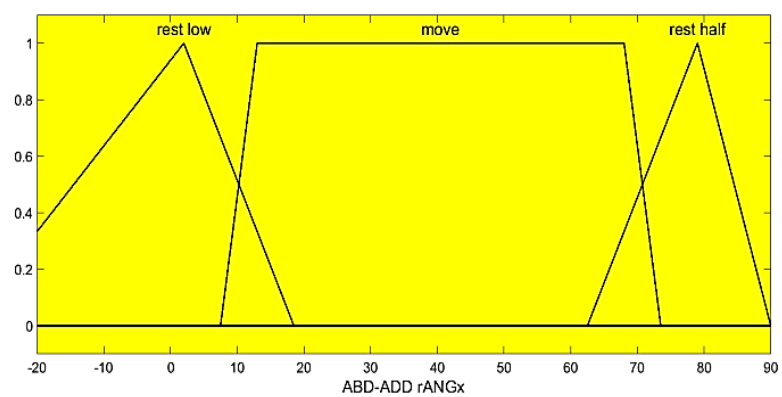

(f) The MFs of rANGx feature

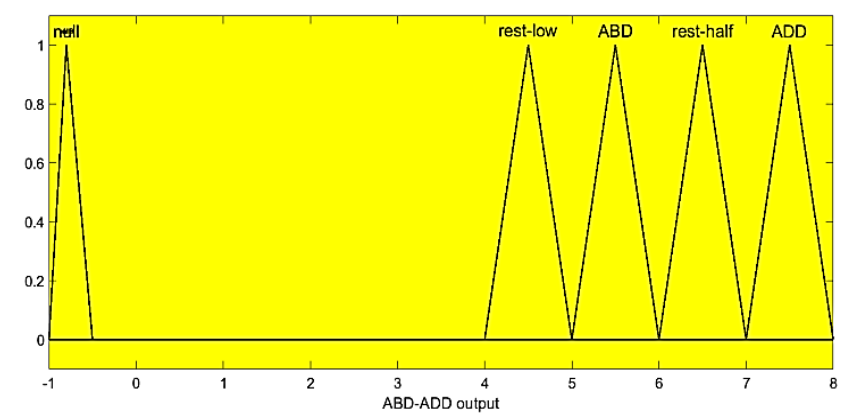

(g) The MFs of ABD-ADD output feature

Fig. 3 Example of data transform between time and frequency domains for the features of the shoulder exercise

(d) We defined the if-then-based Fuzzy rules to control the logic of input and output features in each cyclic epoch. With the rules, the input MFs indicate the consistent output MFs, while the number of the output MFs and the rules must be identical. For an example of the FIS structure of the abd-add motions, we created five dummy rules that represent five virtual motions 
and designed four rules that satisfy four real motions. The initial FIS will be tuned by the ANFIS, which applies the method of Root Mean Square Errors (RMSE) in a data training process, to optimize the MF parameters of the FIS for each epoch.

(e) We defuzzified the trained FIS with the capable MFs and the Fuzzy rules to infer the motion labels in recognition. The process of defuzzification computes participation degrees of the input MFs based on the rules. The test datasets were employed for evaluation. The inferred motion labels were compared with the assigned schedule to evaluate if they are indexed in the expected motion range. We proposed a quartile motion-label scheme to verify the recognition rate of the FIS. The scheme splits the data distribution of motion into four sets (i.e. cut at $25 \%, 50 \%, 75 \%$ ), and it evaluates the accuracy of each quartile set. The sets of $25 \%$ and $75 \%$ are usually counted as changing the motion, while the data set around $50 \%$ (i.e. the middle portion) can be a confidence interval of the reliable motion labels.

Abduction-Adduction Exercise

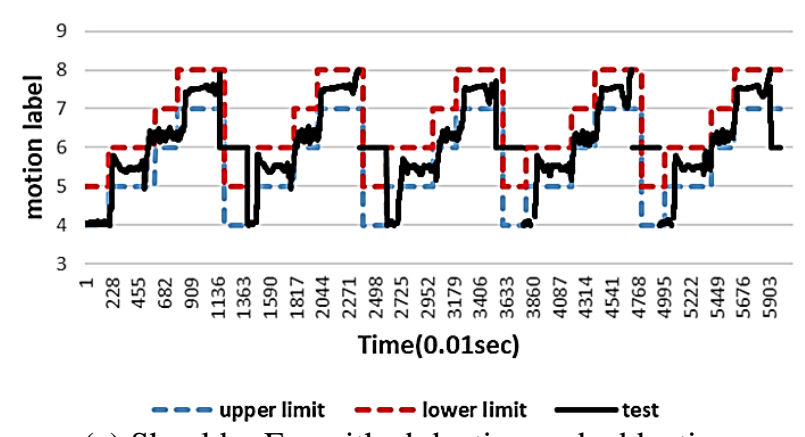

(a) Shoulder Ex. with abduction and adduction
External-Internal Rotation Exercise

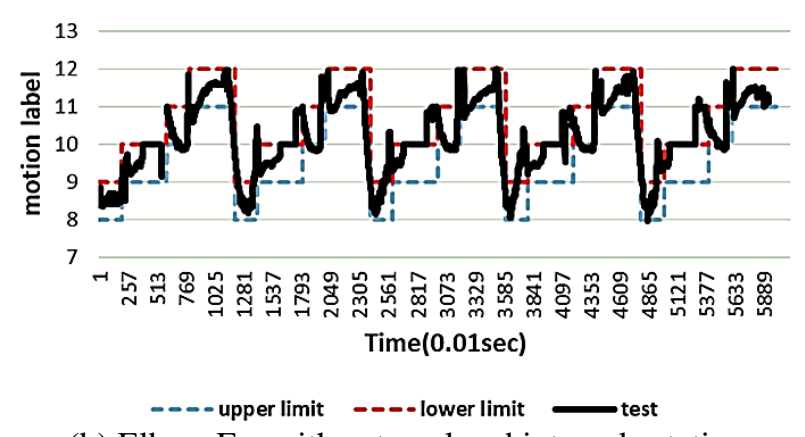

(b) Elbow Ex. with external and internal rotation

Fig. 4 The inference diagrams of the test data versus the sampling schedule

\section{Results and Discussion}

The trained FIS models of the exampled exercises were evaluated by applying the test datasets. The diagrams of both exercises as shown in Fig. 4 compare the labeled test data with the inferred motion labels. The inferred motion labels are observed to fit within the defined range if the motion cycles follow the schedules. The inference diagram displays a visualized chart of both inferred and scheduled motions to track the measurement. When the inferred motion labels were not shown in the range of scheduled motion labels, it would report the outlier movements with respect to the assigned motions. The user is able to obtain a personalized FIS model that is trained by the sample dataset based on the guideline for recognition in measurement. The healthcare professionals can assess rehabilitation performance for improving the therapeutic procedure.

Table 1 The recognition rates based on quartile scheme

for the test data of scheduled exercises

\begin{tabular}{|c|c|c|c|}
\hline \multicolumn{4}{|c|}{ Shoulder Ex with abduction-adduction } \\
\hline motion & $25 \%$ & $50 \%$ & $75 \%$ \\
\hline rest_low & 1 & 0.97 & 1 \\
\hline $\mathrm{ABD}$ & 1 & 1 & 0.8 \\
\hline rest_half & 1 & 1 & 1 \\
\hline ADD & 0.87 & 1 & 1 \\
\hline average & 0.97 & 0.99 & 0.95 \\
\hline \multicolumn{4}{|c|}{ Elbow Ex with external-internal rotation } \\
\hline motion & $25 \%$ & $50 \%$ & $75 \%$ \\
\hline rest_inside & 1 & 1 & 1 \\
\hline ER & 1 & 0.41 & 0.03 \\
\hline rest_outside & 1 & 0.38 & 0.03 \\
\hline IR & 0.71 & 1 & 0.87 \\
\hline average & 0.939 & 0.70 & 0.48 \\
\hline
\end{tabular}

The recognition accuracy of the chosen motions was evaluated by the quartile schemes as shown in Table 1 . The outlier data were aware of moving too fast, which may lead to the angular velocity over the maximum of sample data. The average 
rates of recognition for the Shoulder Ex, which performed steady motions by the schedule, were about 0.99 due to the middle $(50 \%)$ of the quartile scheme. The rates of the Elbow Ex, which did not move stably from external to internal rotation and probably caused data shifts, were ranged from $0.93,0.70$, and 0.48 with respect to $25 \%, 50 \%$, and $75 \%$ portions. This evaluation suggests that the middle of motion data is acceptable for recognition.

We further verified classification performance by combining both exercises and retrieving the middle section of motion data in every second. The subject can randomly choose either shoulder of elbow exercises, but the movement should follow the guide as the best for validation. The sensitivity, specificity, and accuracy of the shoulder exercise are calculated as $0.942,0.998$, and 0.951 , respectively; while they reached $0.995,0.614$, and 0.703 for the elbow exercise.

\subsection{Benefits}

The proposed model follows the process of the supervised algorithm in machine learning to label the motion data based on the scheduled exercise. With the ANFIS, it is available for generating a personalized model of rehabilitation recognition. The proposed Bluetooth-compliant sensor is able to provide plentiful sample datasets in the training procedure. The personal movement features can be chosen to initialize the MFs of FIS with the Fuzzy rules for data training in the ANFIS process. Our approach suggests a confidence interval of the motion to extract proper data, e.g. the middle of each scheduled motion period. The model is achievable for recognizing rehabilitation exercises in healthcare, which is enough for practical application [15].

\subsection{Limitations}

The same feature can measure a variety of exercises within the same range of motions, e.g. the features "rANGVx" and "rANGx" for both of the shoulder and elbow exercises in this study. However, they cannot be combined in an exercise by a unique FIS. Therefore, the user needs to wear more sensors for measuring this complex exercise. In addition, the sensor usually detects unstable noises for irregular motions, which the features are ambiguous in recognition. If the user does not follow the exercise schedule and the tracking data are probably shifted, the measurement is not reliable for the report. In rehabilitation, the physiotherapist used to design simple exercises for the patient.

\section{Conclusions}

We applied the ANFIS to establish a recognition prototype of personalized rehabilitation model for ubiquitous healthcare measurement in this study. The Bluetooth-compliant sensor embedded with the chips of the accelerometer, gyroscope, and inclinometer can be worn to measure the motion data of the rehabilitation exercises. The model presents two exercises with joint-relax motions for the shoulder and elbow. In which, the user wore the sensor on the wrist and obeyed the motion schedule to move the limbs. Each motion dataset is labeled by an arbitrary number within a defined range. An initial FIS with the Fuzzy logic rules was created by the sample datasets; then, the steps of data preprocessing, feature visualizing, fuzzifying, data training, and defuzzifying were processed in the ANFIS. The ANFIS repeated the data training cycles to accomplish the trained FIS, which can defuzzify the test data upon rule-based Fuzzy logics to infer the motion labels and recognize the exercise. In approach, the average recognition rate due to the quartile motion-labels scheme was higher than $90 \%$ if the user faithfully followed the assigned schedule. This approach can be promoted to the UHM for comprehensive health services in the future.

\section{Conflicts of Interest}

The authors declare no conflict of interest.

\section{Acknowledgement}

This work was supported under the Grant MOST 105-2119-M-039-003, 106-2119-M-039-002, 107-2119-M-039-002 by the Ministry of Science and Technology, Taiwan, R.O.C., and the Grant CMU106-S-29 by China Medical University. 


\section{References}

[1] M. Jackson, B. Michaud, P. Tétreault, and M. Begona, "Improvements in measuring shoulder joint kinematics," Journal of Biomechanics, vol. 45, no. 12, pp. 2180-2183, June 2012.

[2] Y. J. Chang, W. Y. Han, and Y. C. Tsai, "A Kinect-based upper limb rehabilitation system to assist people with cerebral palsy," Research in Developmental Disabilities, vol. 34, no. 11, pp. 3654-3659, September 2013.

[3] Darwish and A. E. Hassanien, "Wearable and implantable wireless sensor network solutions for healthcare monitoring," Sensors, vol. 11, no. 6, pp. 5561-5595, May 2011.

[4] M. Chen, S. Gonzalez, A. Vasilakos, H. Cao, and V. Leung, "Body area networks: a survey," Mobile Networks and Applications (MONET). Springer Netherlands. vol. 16, no. 2, pp. 171-193, April 2011.

[5] M. Singh and S. K. Soni, "Spatial correlation-based clustering in wireless sensor network," International Journal of Engineering and Technology Innovation, vol. 8, no. 4, pp. 294-306, January 2018.

[6] P. Mandal, K. Tank, T. Mondal, C. H. Chen, and M. J. Deen, "Predictive walking-age health analyzer," IEEE Journal Biomedical and Health Informatics, vol. 22, no. 2, pp. 363-374, March 2018.

[7] R. R. Fletcher, M. Z. Poh, and H. Eydgahi, "Wearable sensors: opportunities and challenges for low-cost health care," in 2010 Annual International Conference of the IEEE Engineering in Medicine and Biology, Buenos Aires, Argentina, September 2010, pp. 1763-1766.

[8] K. Nguyen-Huu, C. G. Song, and S. W. Lee, "Smartwatch/smartphone cooperative indoor lifelogging system," International Journal of Engineering and Technology Innovation, vol. 8, no. 4, pp. 261-273, September 2018.

[9] C. C. Lee, "Fuzzy logic in control systems: fuzzy logic controller: part II," IEEE Trans. on Systems, Man, and Cybernetics, vol. 20, no. 2, pp. 419-435, 1990.

[10] L. A. Zadeh, "Outline of a new approach to the analysis of complex systems and decision processes," IEEE Transactions on Systems, Man, and Cybernetics, vol. 3, no. 1, pp. 28-44, January 1973.

[11] L. Li, Y. Ma, B. Wang, H. Dong, and Z. Zhang, "Research on traffic signal timing method based on ant colony algorithm and fuzzy control theory," in Proceedings of Engineering and Technology Innovation, vol. 11, pp. 21-29, 2019.

[12] E. H. Mamdani and S. Assilian, “An experiment in linguistic synthesis with a fuzzy logic controller,” International Journal of Man-Machine Studies, vol. 7, no. 1, pp. 1-13, January 1975.

[13] T. Takagi and M. Sugeno, "Derivation of fuzzy control rules from human operator's control actions," Proc. of the IFAC Symp. on Fuzzy Information, Knowledge Representation, and Decision Analysis, Marseilles, France, July 1983, pp. $55-60$.

[14] J. S. R. Jang, “ANFIS: adaptive-network-based fuzzy inference system,” IEEE Transaction on Systems, Man, and Cybernetics, vol. 23, no. 3, pp. 665-685, 1993.

[15] F. Attal, S. Mohammed, M. Dedabrishvili, F. Chamroukhi, L. Oukhellou, and Y. Amirat, "Physical human activity recognition using wearable sensors," Sensors (Basel), vol. 15, no. 12, pp. 31314-31338, December 2015. DOI: $10.3390 / \mathrm{s} 151229858$

Copyright $@$ by the authors. Licensee TAETI, Taiwan. This article is an open access article distributed under the terms and conditions of the Creative Commons Attribution (CC BY-NC) license (https://creativecommons.org/licenses/by-nc/4.0/). 